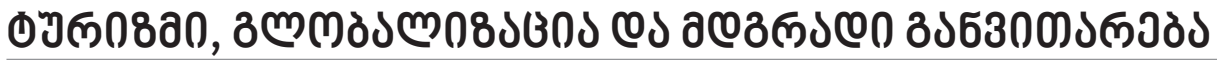

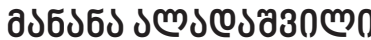

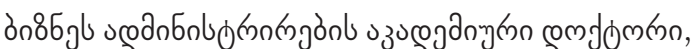

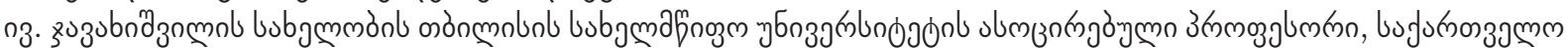

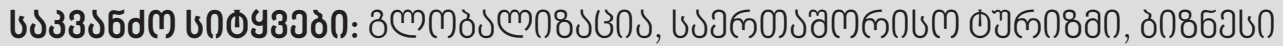

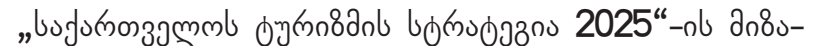

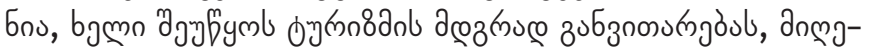

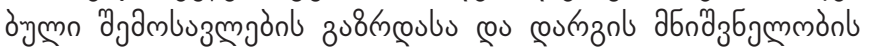
sàmmgoul.

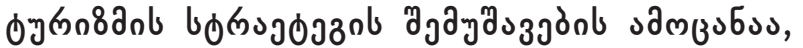

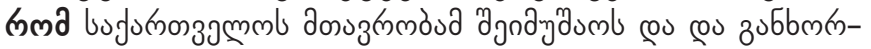

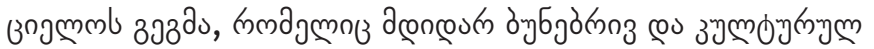

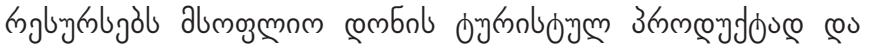

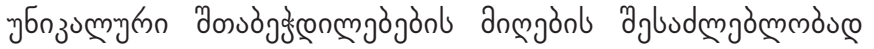

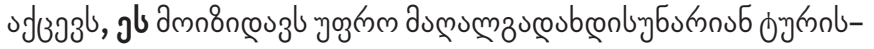

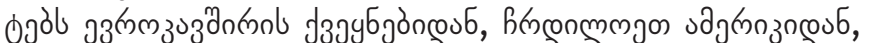

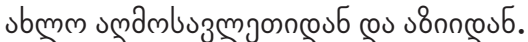

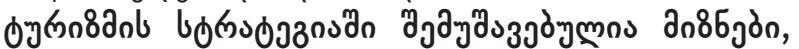

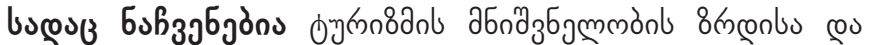

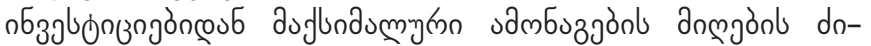

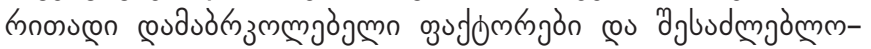

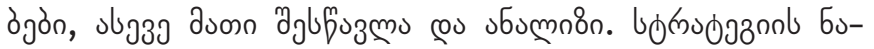

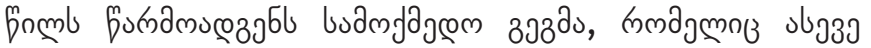

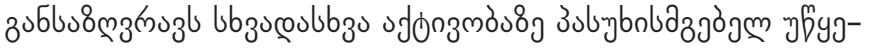

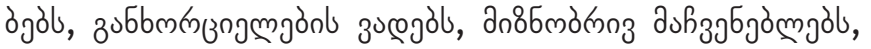

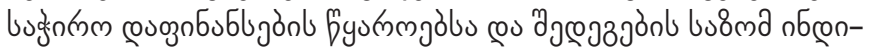

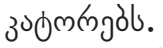

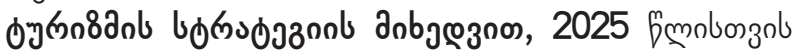

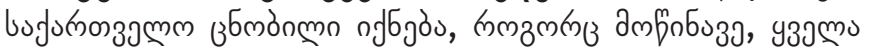

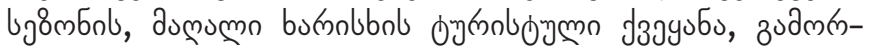

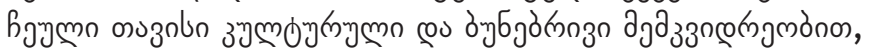

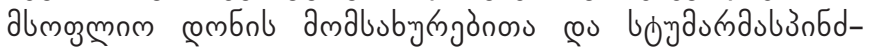

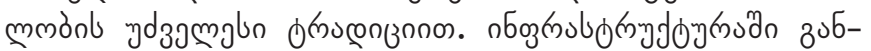

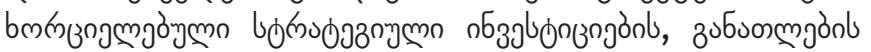

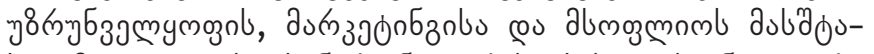

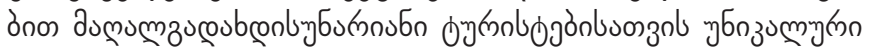

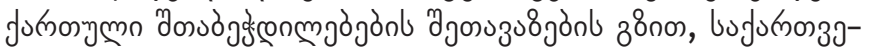

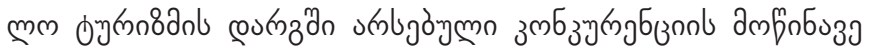

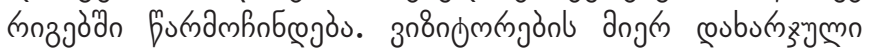

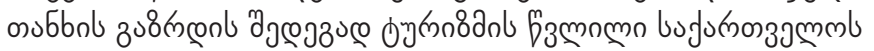

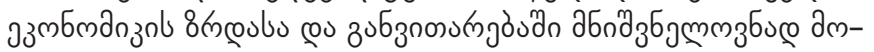

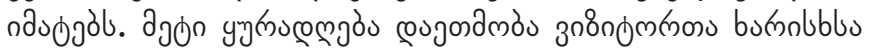

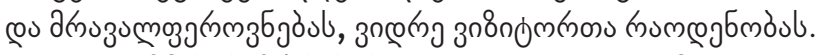

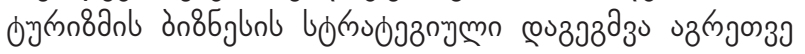

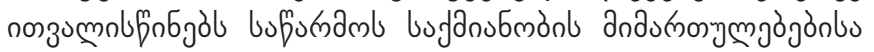

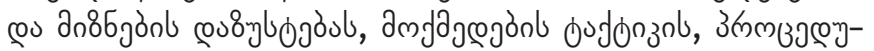

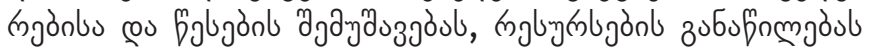

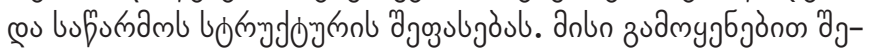
budmgò

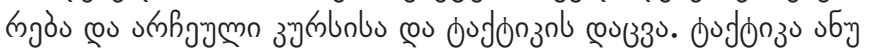

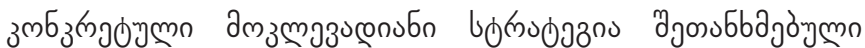

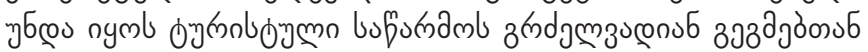

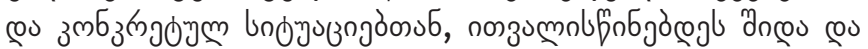

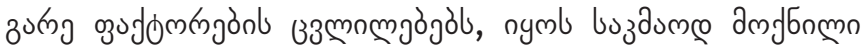

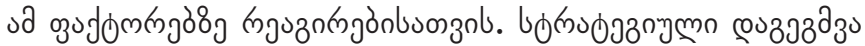

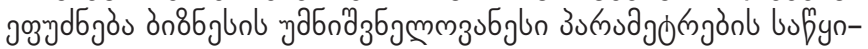

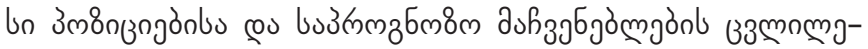

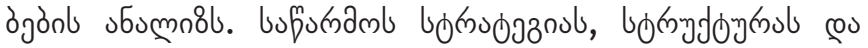

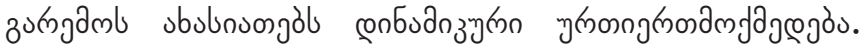

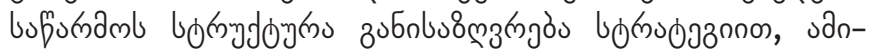

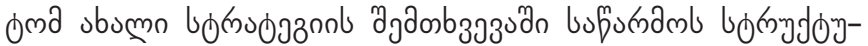

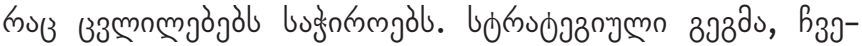

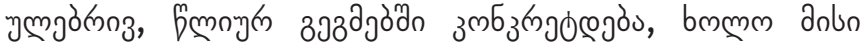

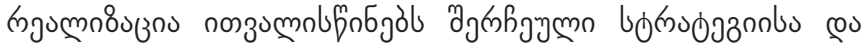

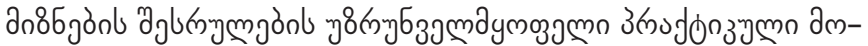

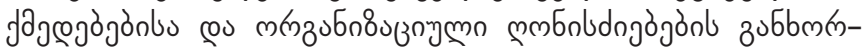
उnjmgoul.

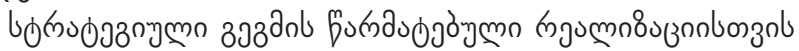
buännmo, फलd:

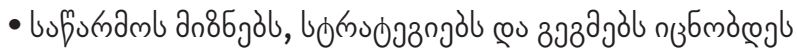

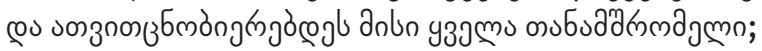

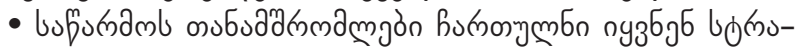

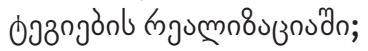

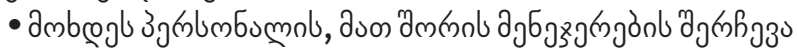

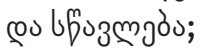

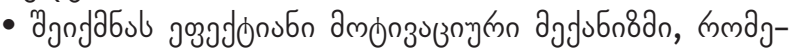

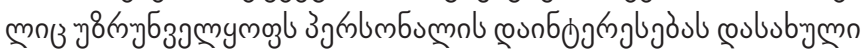

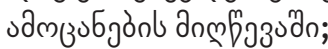

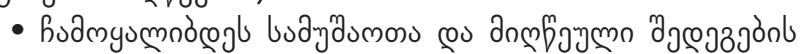

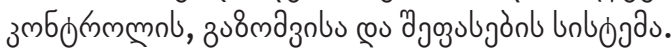

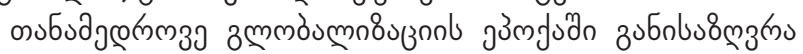

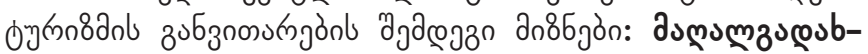

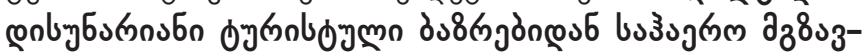

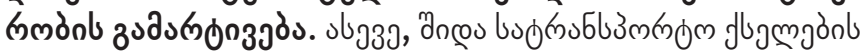

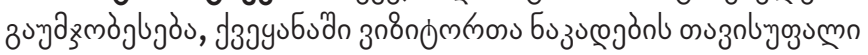

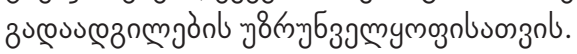

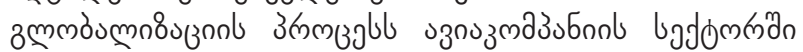

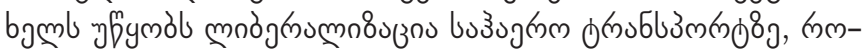

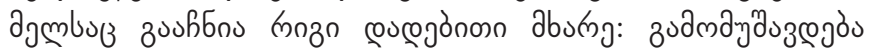

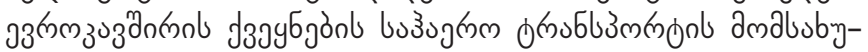

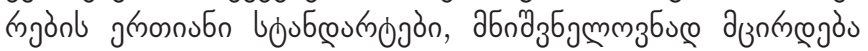

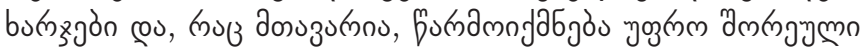

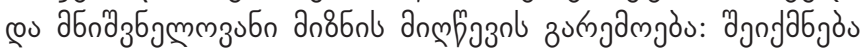

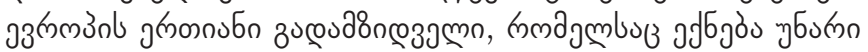

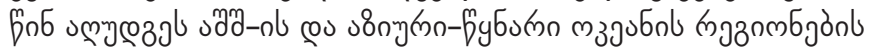




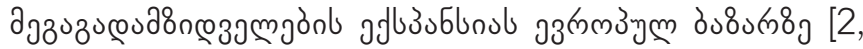
33. 40].

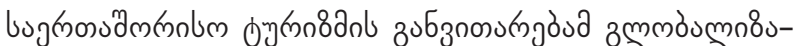

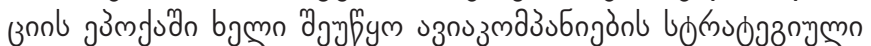

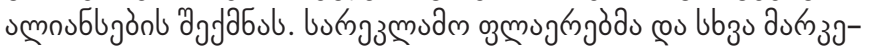

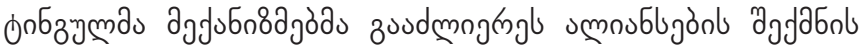

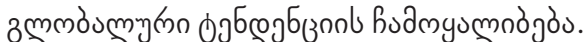

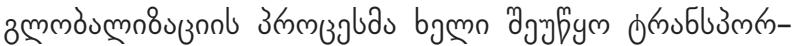

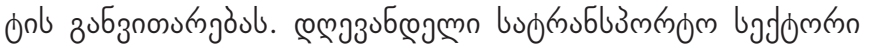

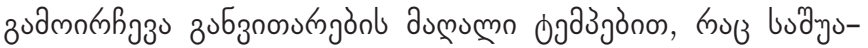

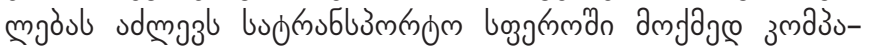

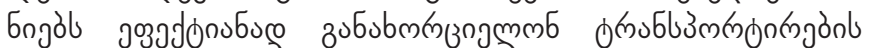

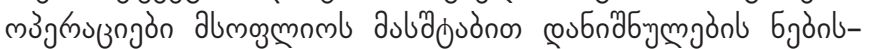

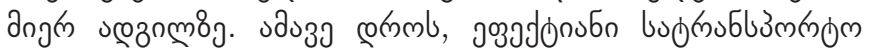

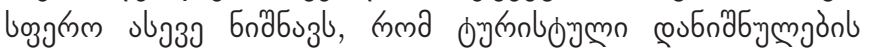

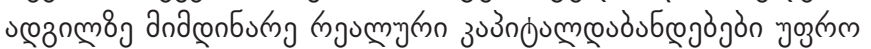

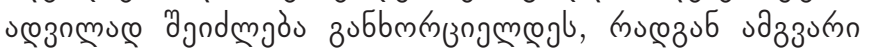

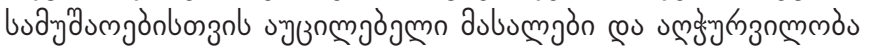

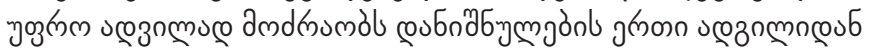

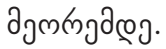

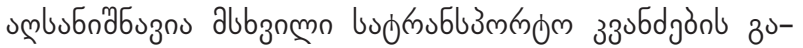

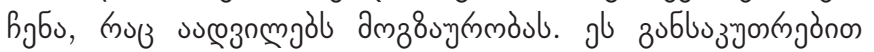

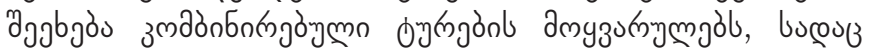

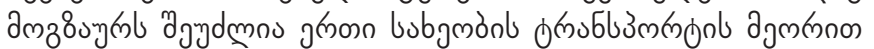

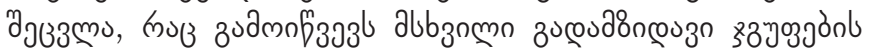

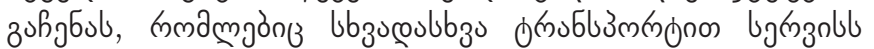

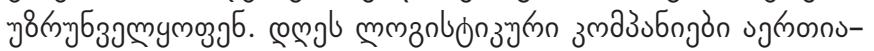

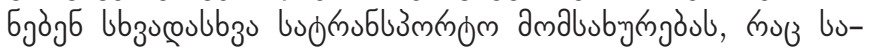

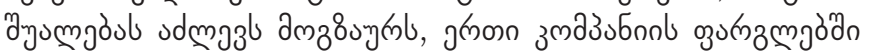

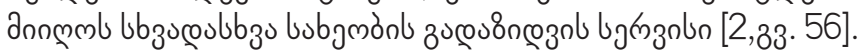

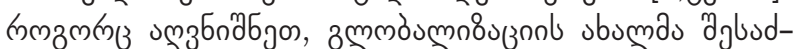
млдेмm

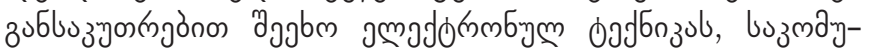

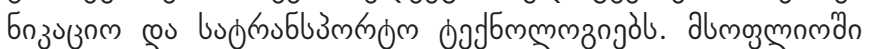

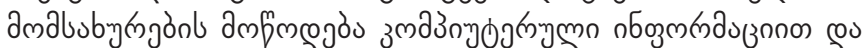

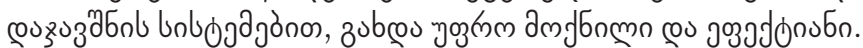

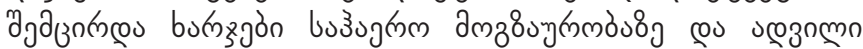

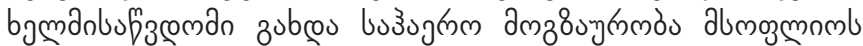

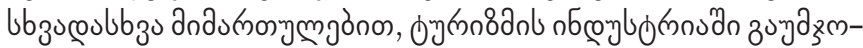

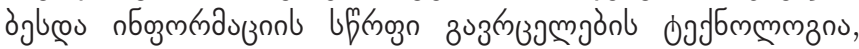

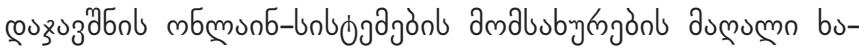

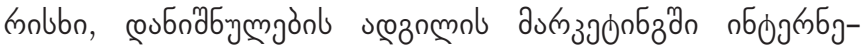

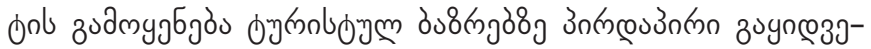

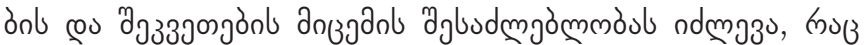

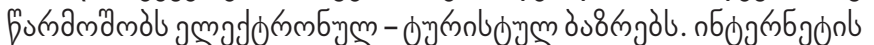

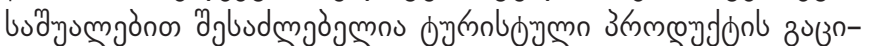

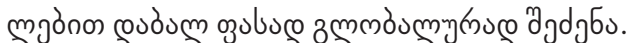

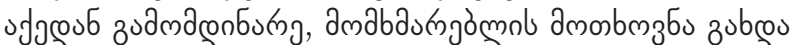

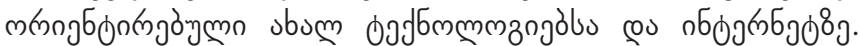

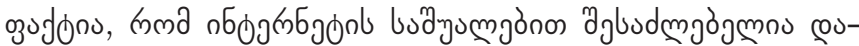

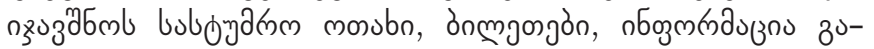

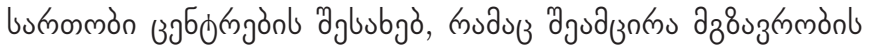

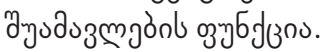

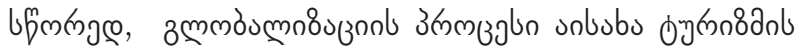

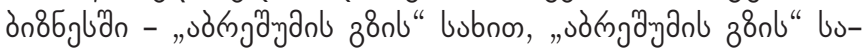

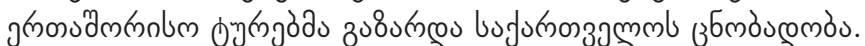

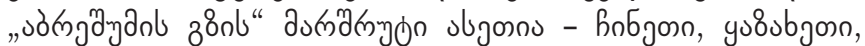

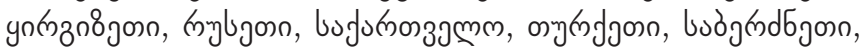

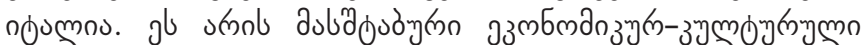

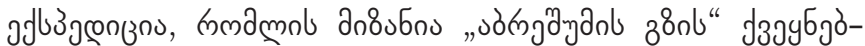

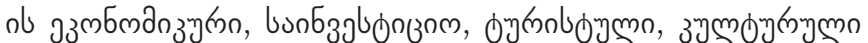

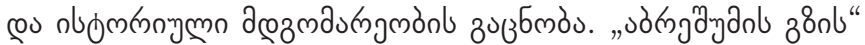

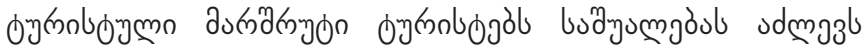

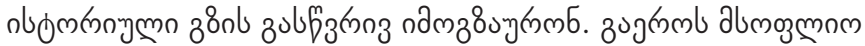

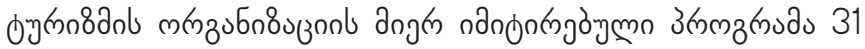

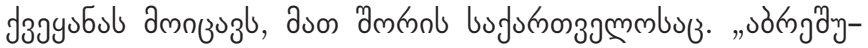

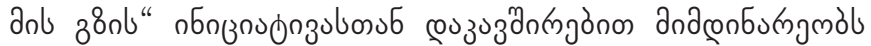

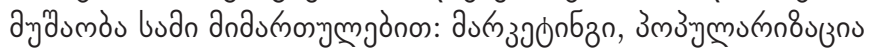

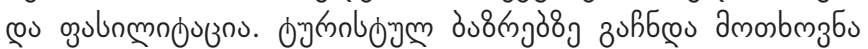

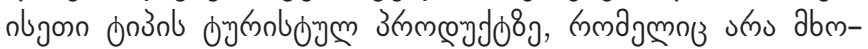

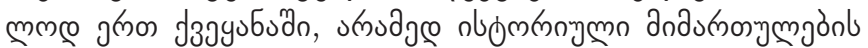

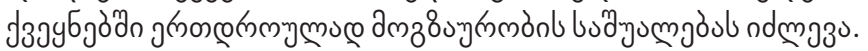
[4]

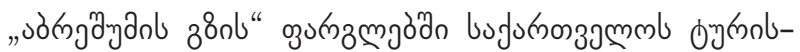

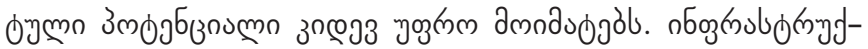

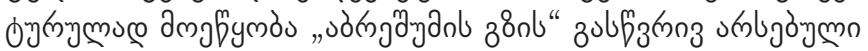

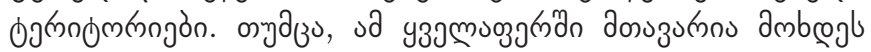

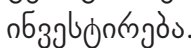

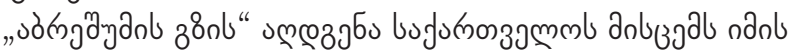

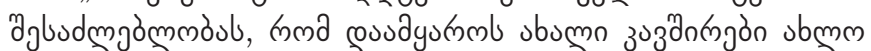

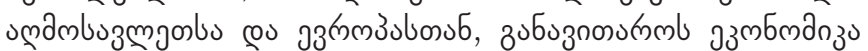

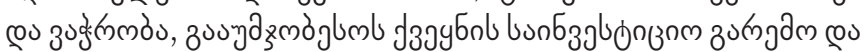

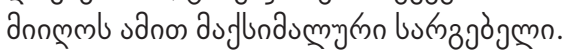

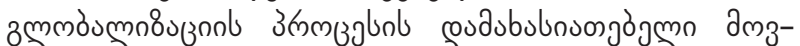

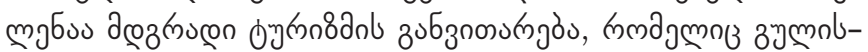

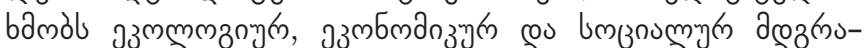

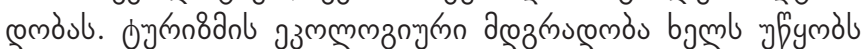

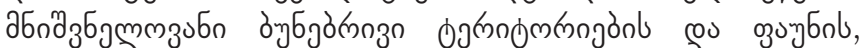

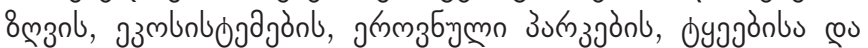

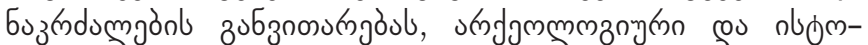

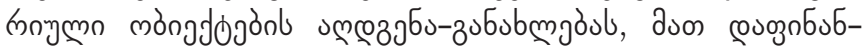

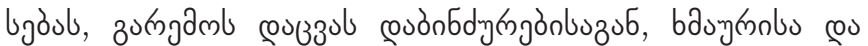

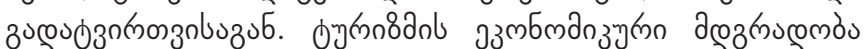

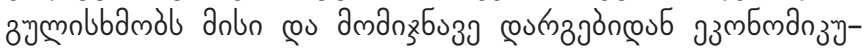

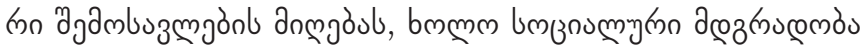

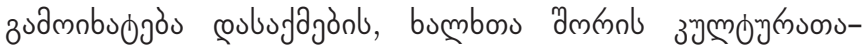

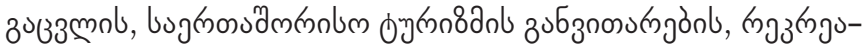

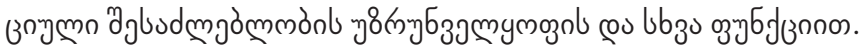

з мmलठumo

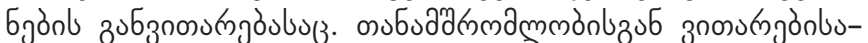
зj б

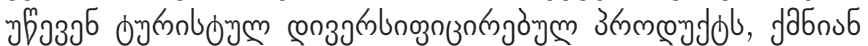

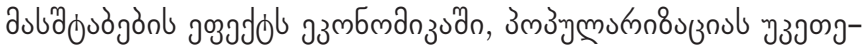

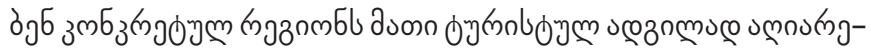
onb 38 กnom. [3, 33.15]

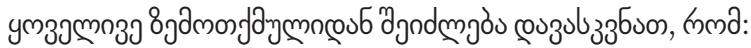




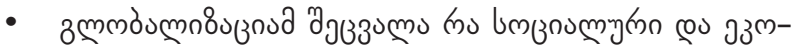

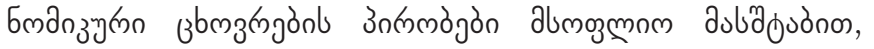

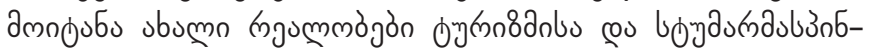

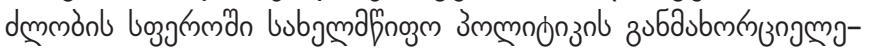

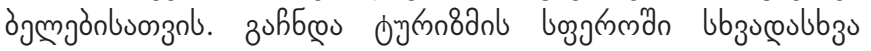

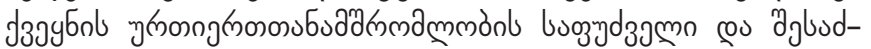

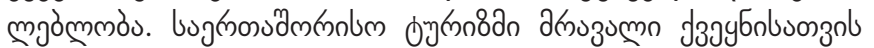

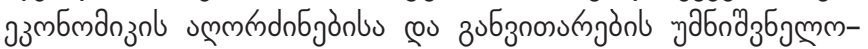
zubju oु०dommoco nflo;

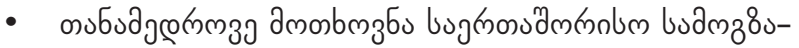

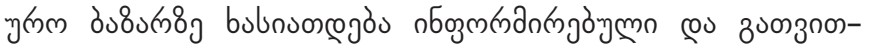

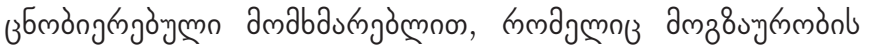

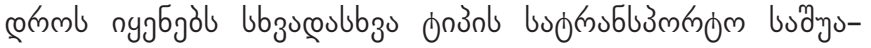

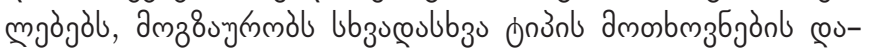

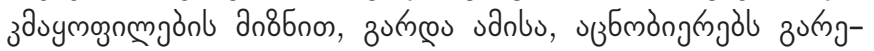

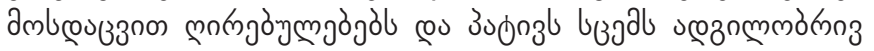
उचmognol;

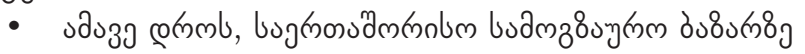

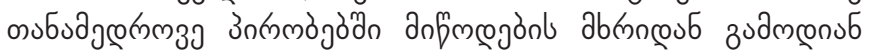

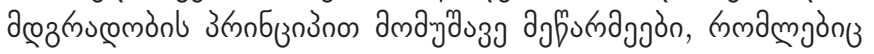

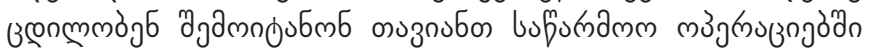

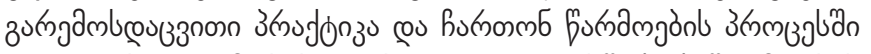

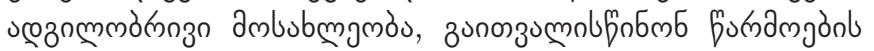

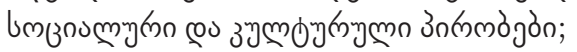

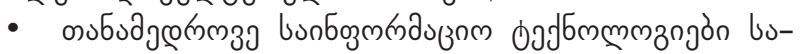

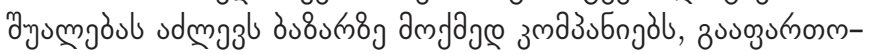

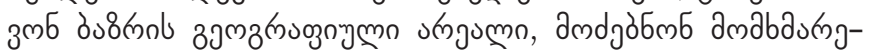

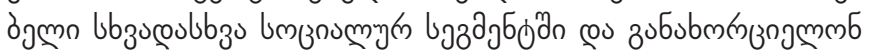

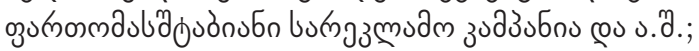

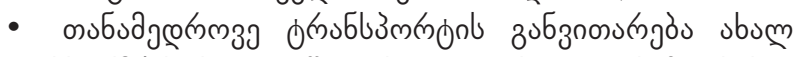

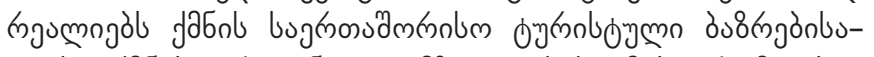

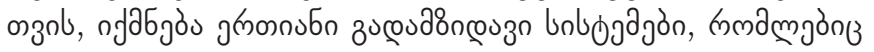

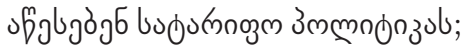

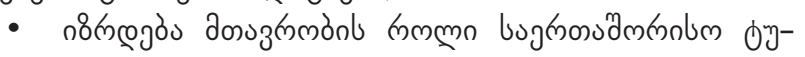

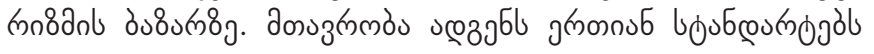

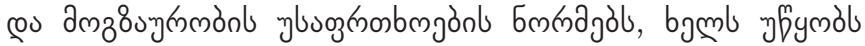

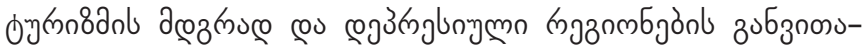
ngouls.

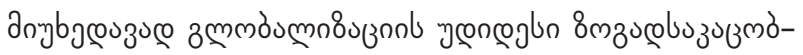

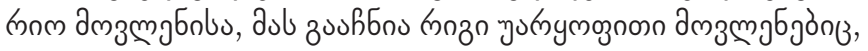

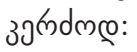

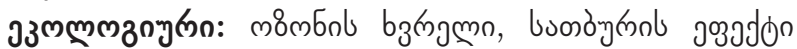

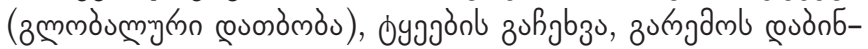

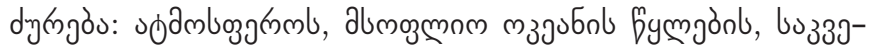

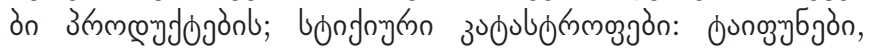

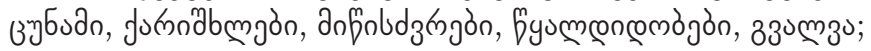

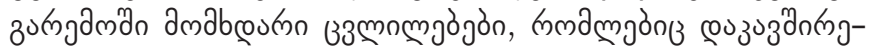

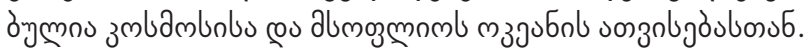

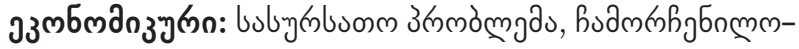

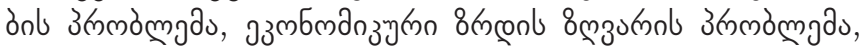

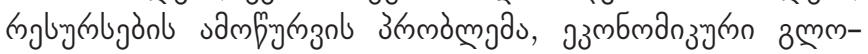

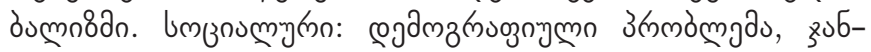

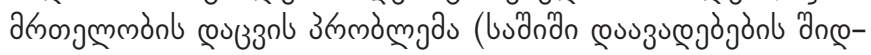

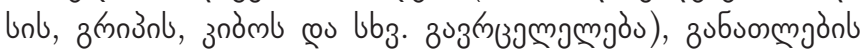

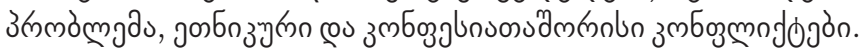

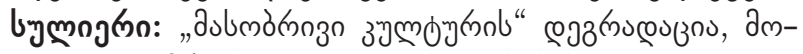

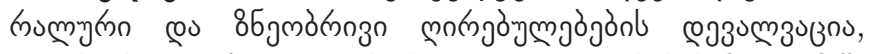

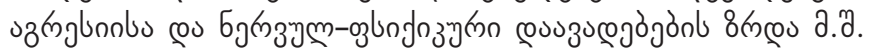

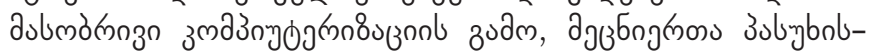

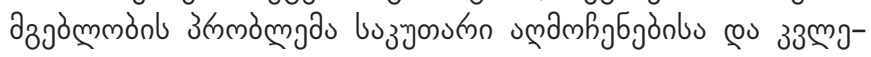
उgòn zudm.

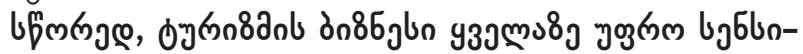

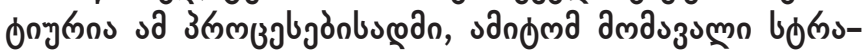

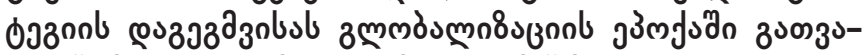

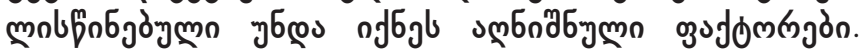

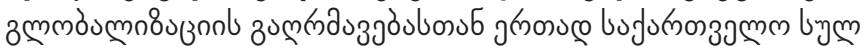

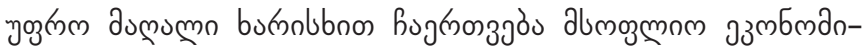

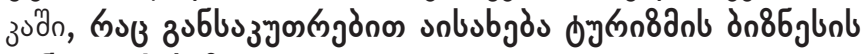

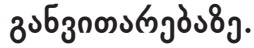

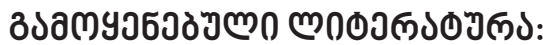

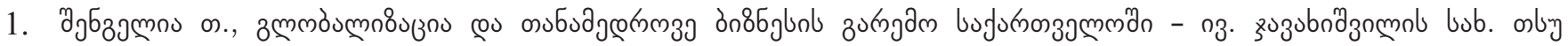

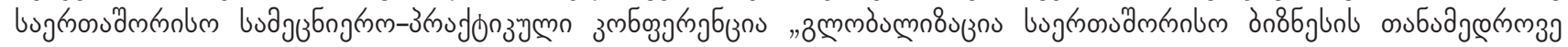

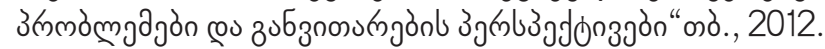

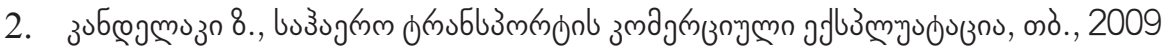

3. Iris Mihailovic - University od Dubrovnik, Department of Economics and Business Economics, Croatia - European scientific Journal, August 2014.

4. UNWTO Touriam Higlights 2015 edition .

5. WTTC Travel and Tourism. World economic impact 2015

6. Петрасов И., Концепция устойчивого развития применительно к мировому туризму (www.geopub.narod.ru).

7. Назачук А. В., Этика глобализирующегося общества (www.ihtik.lib.ru).

8. Покровский Я.Е., Транзит российскихценностей:нереализованная альтернатива, аномия, глобализация. 2000 (www.i-u.ru/biblio/archive/novikova_soc/soc_nov22.aspx). 


\title{
TOURISM, GLOBALIZATION AND SUSTAINABLE DEVELOPMENT
}

\author{
MANANA ALADASHVILI
}

https://doi.org/10.35945/gb.2017.03.022

Doctor of business administration,

Associated Professor of Ivane Javakhishvili Tbilisi State University, Georgia

KEYWORDS: TOURISM, GLOBALISATION, SUSTAINABLE DEVELOPMENT

\section{SUMMARY}

In contemporary terms, tourism is a highly complex, multifunctional, multidimensional, and dynamic category of national and international significance. Therefore, it is necessary to primarily consider and analyse the very concept of tourists and tourism; its essence, structure, types and forms in which it primarily exists as a social, cultural and economic phenomenon, as a practice and a science. Globalisation is a relatively new concept in economics, and it refers to the opening of individual national economies to the world economy, with mutual strong co-operation with as few legal barriers as possible. Sustainable development is also a recent economic concept, and it refers to the adjustment of economic development of a certain country to its natural resources, but also the resources of other countries that the respective country uses for its economic activities. The abovementioned differences in the outlook on tourism, globalisation and sustainable development indicate that tourism is a very heterogeneous social phenomenon, and that different approaches and conclusions arise from these differences. Such activity has prompted the need for better linking of economic and political systems of different countries. It is indubitable that the boost of contemporary globalisation is related to economic growth and depletion of natural resources at the global level. This indicates that the globalisation process in terms of energy and environmental constraints could have a self-annihilating character. Simultaneous effects of tourism, globalisation process and sustainable development will significantly affect future development of overall economic and social changes worldwide. 